\title{
Modelagem e otimização de um reator trifásico de leito fixo gás-líquido-sólido (Trickle Bed) para in- dústria do Petróleo usando a técnica numérica transformada diferencial
}

Santana, C. V.

Escola Politécnica de Pernambuco Universidade de Pernambuco 50.720-001 - Recife, Brasil jornadesdias@poli.br
Silva, J. D.

Escola Politécnica de Pernambuco

Universidade de Pernambuco

50.720-001 - Recife, Brasil

Resumo O reator de leito fixo (trickle bed) tem sido amplamente estudado atualmente devido a exigências de um maior controle na emissão de poluentes na indústria do petróleo. Nesse intuito esse trabalho tem como foco a exposição do funcionamento deste reator trifásico bem como otimização do mesmo através de modelos matemáticos dinâmicos gerais e simplificadores que possam fornecer de forma segura e confiável os resultados da fenomenologia realista do regime de escoamento. Palavras chave: leito fixo, modelagem, otimização.

Abstract

The fixed bed reactor (trickle bed) has been extensively studied currently due to demands for a better control of the emission of pollutants in the oil industry. This project has as a goal explain the operation of this three-phase reactor both optimization through general and simplifiers dynamic mathematical models that can provide safely and reliably the results of phenomenology realistic of the flow regime. 


\section{Introdução}

Aspectos dinâmicos relacionados à análise de sistemas trifásicos com contato fluido-sólido têm recebido grande atenção, tendo em vista as possibilidades da quantificação dos fenômenos ocorrendo nas operações de sistemas gáslíquido-sólido. Fenômenos de transferência de massa gáslíquido, líquido-sólido e gás-sólido, dispersão axial, difusão intraparticular, adsorção e reação na superfície catalítica podem ser quantificados através de parâmetros representativos e incluídos nos modelos que venham a descrever o comportamento dos sistemas trifásicos gás-líquidosólido. Complementações a modelagem podem ser introduzidas, fruto de extensivos trabalhos experimentais, fornecendo correlações, parâmetros adimensionais e mapeando regiões de operações relativas ao escoamento de fluidos em contato com os sólidos. A descrição completa do comportamento de operação de processos em reatores trifásicos de leito fixo sob regime de leito gotejante, com escoamento concorrente descendente das fases gasosa e líquida sobre partículas sólidas, envolve aspectos relacionados à hidrodinâmica das fases fluidas, transferência de massa interfases gás-líquido, gás-sólido e líquido-sólido, à difusão nos poros do catalisador e às interações fluido-sólido como adsorção, reação e dessorção. Enfatizando a análise desses reatores trifásicos, recorre-se à aplicação de modelos dinâmicos capazes de descrever a fenomenologia realista dos regimes transiente e estacionário, permitindo avaliações seguras e confiáveis em controle e otimização do processo [1],[2],[3].

\section{Modelagem Matemática}

Com o objetivo de descrever de forma simplificada os processos que ocorrem na região de contato do Reator Trifásico de Leito Fixo, este foi modelado e simulado utilizando artifícios matemáticos. Métodos de diferenciação foram aplicados às equações diferenciais. Os modelos encontrados podem ser depois devidamente comparados com dados disponíveis na literatura. Para o modelo desenvolvido considera-se uma geometria cilíndrica, considerando o efeito de distribuição (alimentação na base do reator e produtos retirados no topo). A pesquisa que visa melhorar o desempenho dos reatores de leito gotejante tem sido estimulada principalmente pela necessidade do mercado de derivados do petróleo, buscando maiores quantidades de produtos de frações leves de petróleo em refinarias. Desta forma, o modelo matemático abaixo é destinado para esta pesquisa.

- Balanço de energia para a fase gasosa;

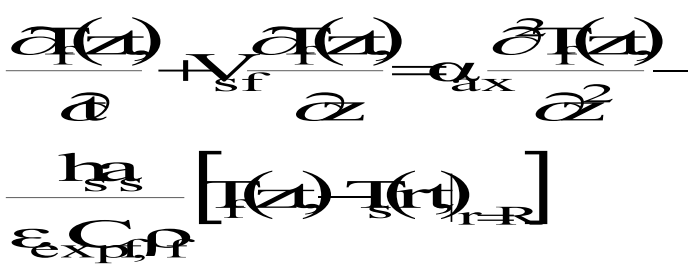

Condições iniciais e de contorno para a Equação (1);

$\mathbf{T}(\mathbf{t})_{\mathbf{t}=\mathbf{O}}=\mathrm{T}_{\mathrm{fo}} ; 0 \square \mathrm{z} \square \mathrm{L}$ des iniciais e de

contorno para a Equação (53):

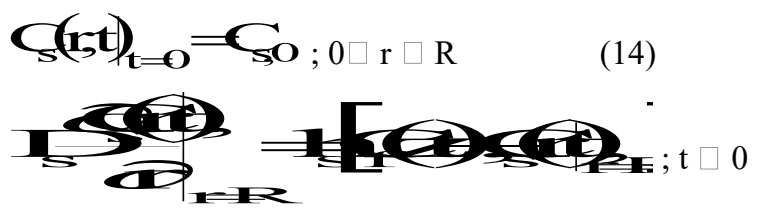

(15)

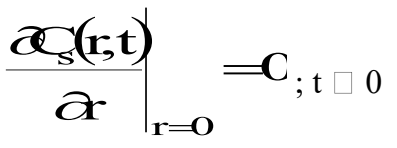

Balanço de massa para adsorção:

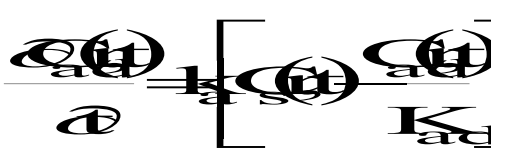

Condições iniciais e de contorno para a Equação:

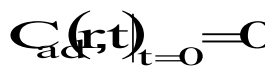

\section{Resultados}

Através da discretização das equações de balanço, foram desenvolvidas equações iterativas para as temperaturas das fases gasosa e sólida e para as concentrações das fases gasosa, sólida e de adsorção. Tais equações seriam:

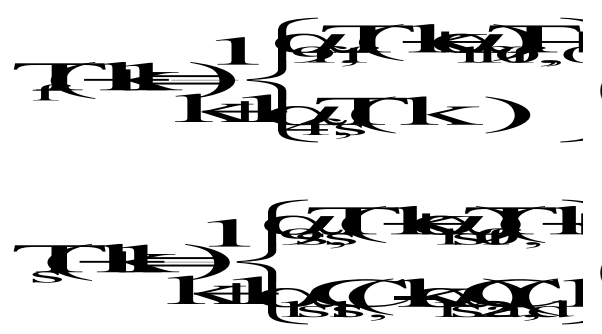



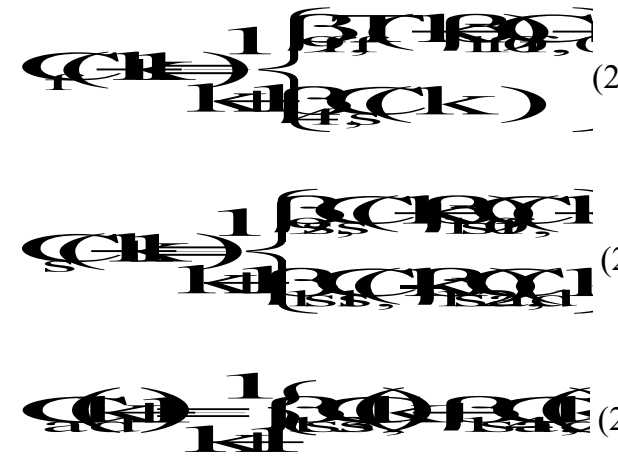

Para a implementação do método foram feitas duas considerações matemáticas. Os valores de $\Delta z$ (que corresponde a variação da distância axial do reator) e $\Delta r$ (variação da distância radial da partícula) foram representados com os valores 0,1 e 0,05 respectivamente. Aplicando iterações sucessivas (um valor total de 300 iterações para cada variável), foram obtidos os seguintes resultados:

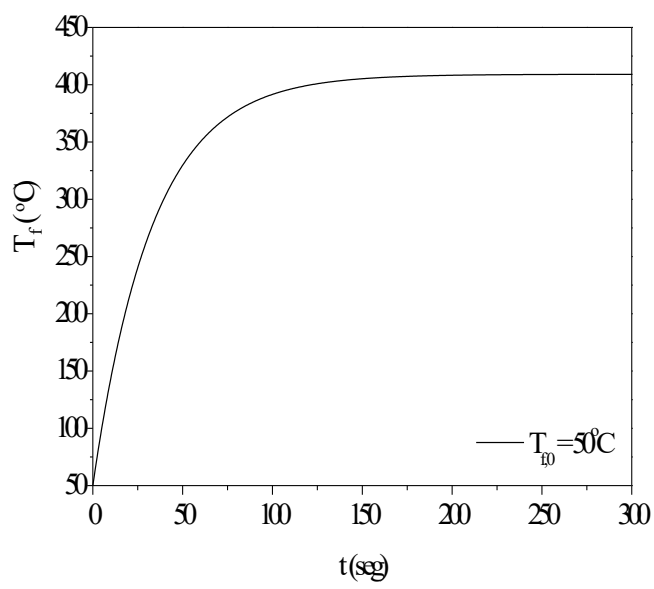

Fig 1. Comportamento da Temperatura da Fase Gasosa como Função do Tempo com uma Velocidade Superficial do Fluido, Vsf = 2,94x10-2 m s-1.

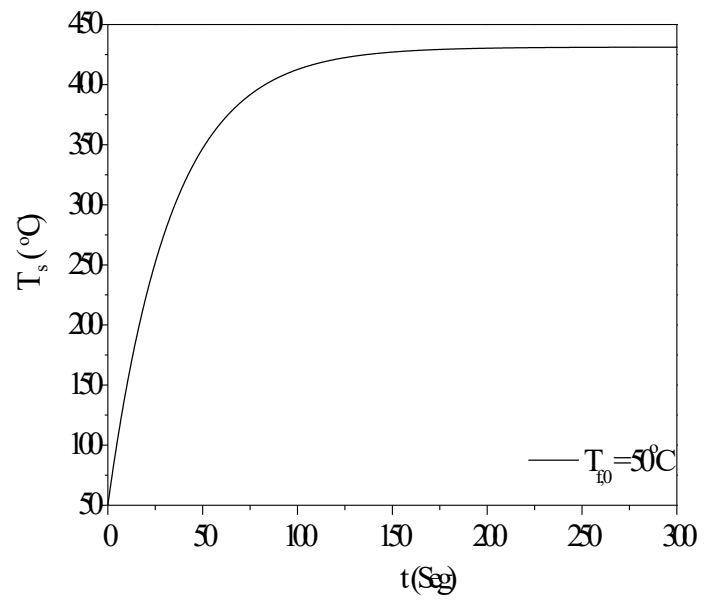

Fig 2. Comportamento da Temperatura da Fase Sólida como Função do Tempo com uma Velocidade Superficial do Fluido, Vsf $=2,94 \times 10-2 \mathrm{~m} \mathrm{~s}-1$.

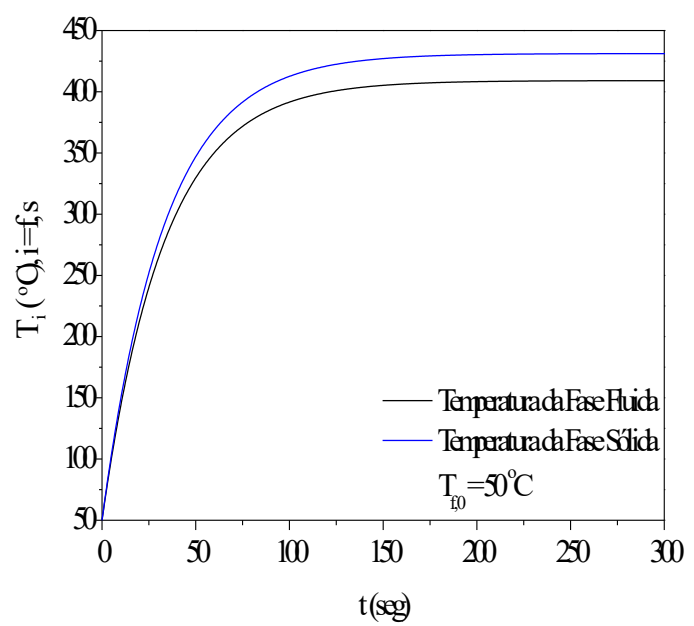

Fig 3. Comparação entre as Temperaturas da Fase Fluida e Sólida como Função do Tempo com uma Velocidade Superficial do Fluido, Vsf $=2,94 \times 10-2 \mathrm{~m} \mathrm{~s}-1$. 


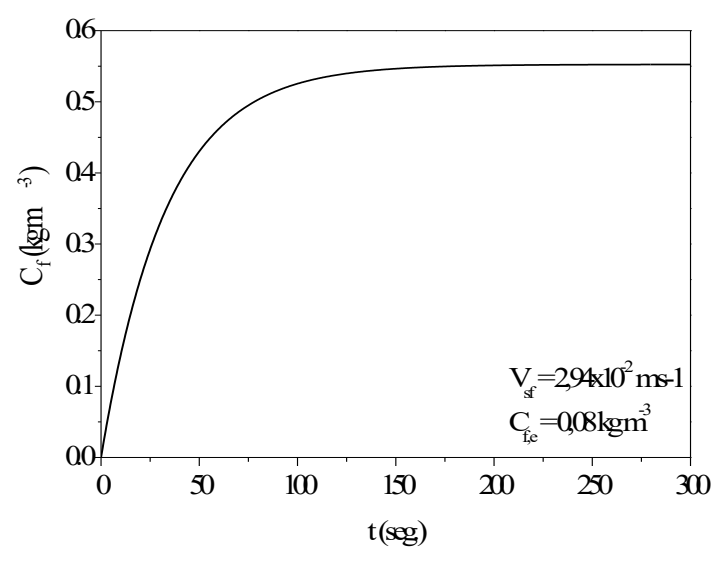

Fig 4. Comportamento da Concentração da Fase fluida como Função do Tempo com Temperatura de Entrada de $\mathrm{Tf}=50^{\circ} \mathrm{C}$.

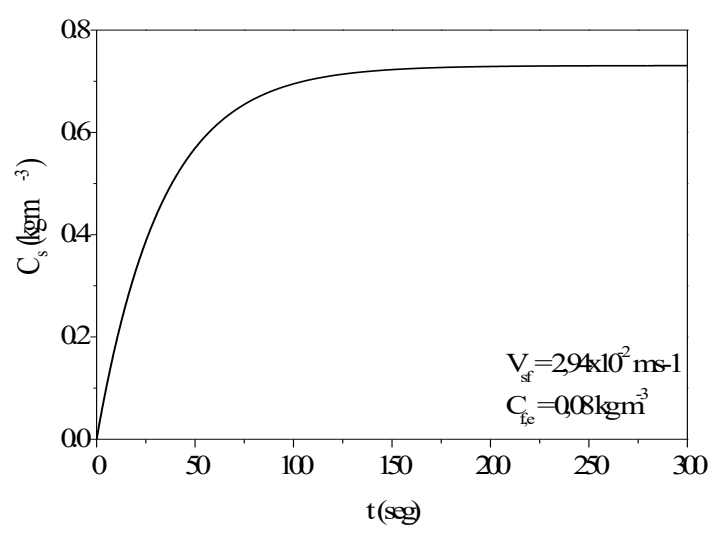

Fig 5. Comportamento da Concentração da Fase Sólida como Função do Tempo com Temperatura de Entrada de $\mathrm{Tf}=50^{\circ} \mathrm{C}$.

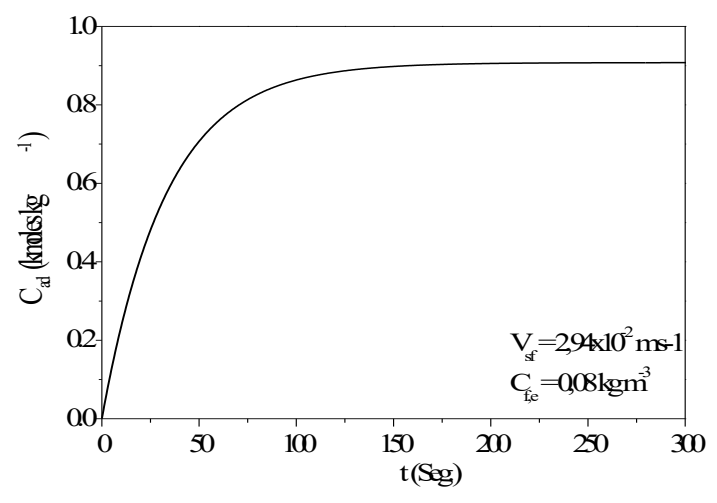

Fig 6. Comportamento da Concentração de Adsorção na Sólida como Função do Tempo com Temperatura de Entrada de $\mathrm{Tf}=50^{\circ} \mathrm{C}$.

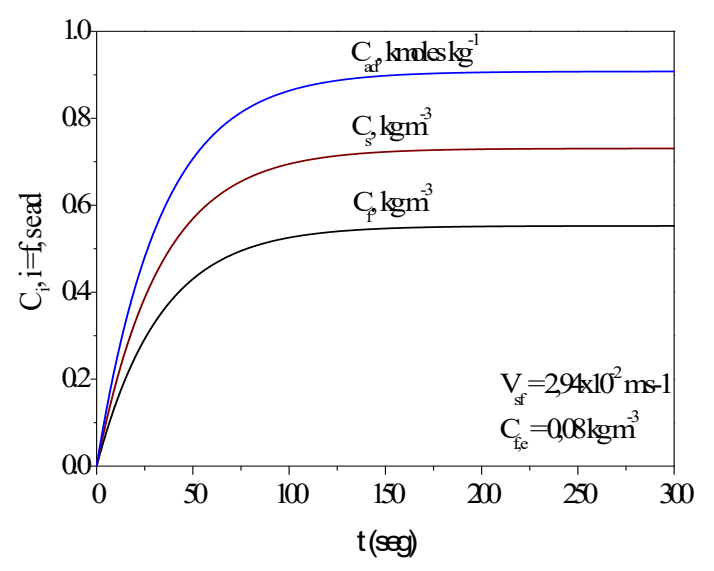

Fig 7. Comparação das concentrações das fases gasosa, sólida, e de concentração de adsorção como Função do Tempo com Temperatura de Entrada de $\mathrm{Tf}=50^{\circ} \mathrm{C}$.

\section{Conclusões}

Foram obtidos os seguintes resultados tomando por base os estudos, modelagem e simulação dos balanços de energia e massa de um reator trifásico de leito fixo: 1) Análise do comportamento das temperaturas de ambas as fases gasosa e sólida; 2) Observação do comportamento das concentrações das fases sólida e líquida de um processo catalítico bem como da concentração de adsorção.

A elaboração do código computacional para processar e analisar o comportamento das variáveis em estudo desta pesquisa permitiu a conduzir as seguintes conclusões: 
- Analisar os comportamentos das temperaturas nas fases gasosa e sólida bem como uma análise comparativa entre as temperaturas das duas fases;

- Observar os comportamentos das concentrações nas fases fluida e sólida como também a concentração do componente adsorvido nos poros da fase líquida. Além disso, também foi observado, usando uma análise comparativa, evolução das concentrações nas fases fluida e sólida bem como a concentração do componente adsorvido.

\section{Anotações}

$\mathrm{C}_{\mathrm{f}} \quad$ Concentração da fase fluida, $\mathrm{kg} / \mathrm{m}^{3}$

$\mathrm{C}_{\mathrm{s}} \quad$ Concentração da fase sólida, $\mathrm{kg} / \mathrm{m}^{3}$

$\mathrm{C}_{\mathrm{ad}} \quad$ Concentração adsorvida, mole $/ \mathrm{kg}$

$\mathrm{C}_{\mathrm{f}, 0} \quad$ Concentração inicial da fase gasosa, $\mathrm{kg} / \mathrm{m}^{3}$

$\mathrm{Cs}, 0$ Concentração inicial da fase sólida, $\mathrm{kg} / \mathrm{m}^{3}$

$\mathrm{K}_{\mathrm{ad}} \quad$ Constante de adsorção, $\mathrm{m}^{3} / \mathrm{kg}$

$\mathrm{T}_{\mathrm{f}, 0}$ Temperatura inicial da fase gasosa, $\mathrm{K}$

$\mathrm{C}_{\mathrm{ad}, 0}$ Concentração inicial de adsorção, $\mathrm{kg} / \mathrm{m}^{3}$

$\mathrm{V}_{\text {sf }} \quad$ Velocidade superficial do fluido, $\mathrm{m} / \mathrm{s}$

$\alpha_{\alpha x}, \quad$ Dispersão da fase térmica

\section{Agradecimentos}

Gostaríamos de agradecer pelo apoio financeiro à Escola Politécnica de Pernambuco que permitiu integralmente a realização da pesquisa no Laboratório de Tecnologia Energética e Ambiental - LATEA

\section{Referências}

[1] Iliuta, I.; Bildea, S. C.; Iliuta, M. C.; Larachi, F. Analysis of Trickle Bed and Packed Bubble Column Bioreactors for Combined Carbon Oxidation and Nitrification. Brazilian Journal of Chemical Engineering. 19: 69-87, 2002.

[2] Silva, J. D. Dynamic evaluation for liquid tracer in a trickle bed reactor. J. Braz. Soc. Mech. Sci. \& Eng., v. 33, n. 3, 2011. DOI: 10.1590/S167858782011000300002

[3] Silva, J.D.; Lima, F.R.A.; Abreu, C.A.M. and Knoechelmann, A. Experimental analysis and evaluation of the mass transfer process in a trickle-bed reactor. Braz. J. Chem. Eng., 2003, vol.20, n.4, pp. 375-390. DOI: 10.1590/S010466322003000400005 .
[4] DECKWER, W.-D. Bubble column reactors. New-York: John Wiley, 1992.

[5] WESTERTERP, K. R, van SWAAIJ, W. P. M., BEENACKERS, S. A C N. Chemical reactor design and operation. New York: John Wiley \& Sons, 1984.

[6] DORAISWANY, L. K, SHARMA, M. M. Heterogeneous reactions - analysis, examples, and reactor design. New York: John Wiley \& Sons, v.2, 1984.

[7] HAN, S., ZHOU, J., JIN, Y., LOH, K C., W ANG, Z. Liquid dispersion in gas-liquid-solid circulating fluidized beds. Chem. Eng. J., v.70, p.914, 1998. 